Erratum

\title{
The Role of CHK1 Varies with the Status of Oestrogen- receptor and Progesterone-receptor in the Targeted Therapy for Breast Cancer: Erratum
}

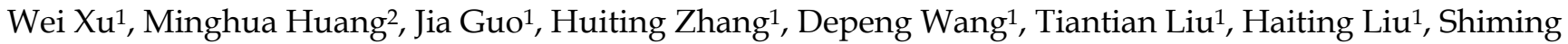
Chen', Peng Gao, ${ }^{1,3}$, Kun $\mathrm{Mu}^{1,3}$

1. Department of Pathology, School of Basic Medical Sciences, Shandong University, Jinan, 250012, China.

2. Department of Respiratory and Critical Care Medicine, The second affiliated Hospital of Nanchang University, Nanchang, 330006, China.

3. Department of Pathology, Qilu Hospital, Shandong University, Jinan, 250012, China.

$\triangle$ Corresponding author: Correspondence and requests for Methodology and Materials should be addressed to Kun Mu; Department of Pathology, Qilu Hospital, Shandong University, Wen Hua Xi Road 44, Jinan, 250012, China; Fax: 86-531-88383168; Email: mukun@sdu.edu.cn.

(c) The author(s). This is an open access article distributed under the terms of the Creative Commons Attribution License (https://creativecommons.org/licenses/by/4.0/). See http://ivyspring.com/terms for full terms and conditions.

Published: 2021.02 .20

Corrected article: Int J Biol Sci 2020; 16(8): 1388-1402. doi: 10.7150/ijbs.41627.

Recently, we have found a mistake in Figure 3I of our paper[1]. In the original manuscript submitted to reviewers, the content of Figure 3I is correct. However, in the process of marking the molecular weight of the indicated proteins according to reviewers' comments, the content of the Figure 3I was completely covered by Figure 3F due to our operational mistake. It should be emphasized that this mistake occurred in the typesetting process of Figure 3 and did not affect the research results and conclusion of this article. Here, Figure 3 has been presented as follow with the corrected Figure 3I, and there is no need to change the legend. All authors have agreed to the Erratum, and we apologize for the negligence in our work and hope to get the opportunity to correct this mistake. 


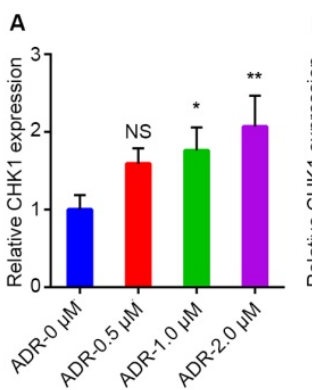

E

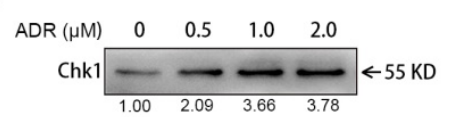

Chk1 (Ser317) $=-55$ KD

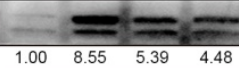

Chk1 (Ser345) $\square-\square 5 \mathrm{KD}$

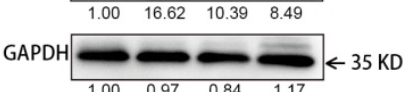

B
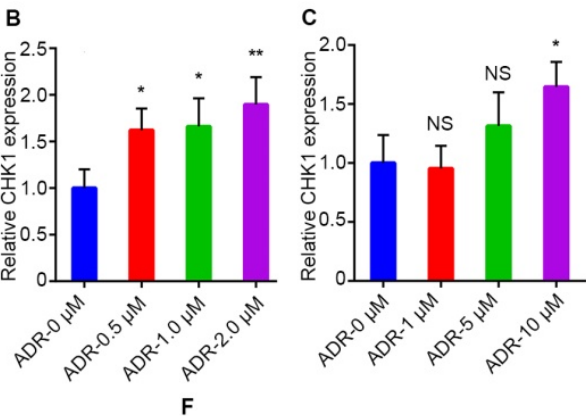

$\operatorname{ADR}(\mu \mathrm{M})$

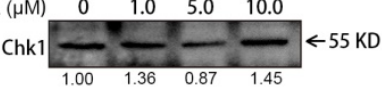

Chk1 (Ser317)
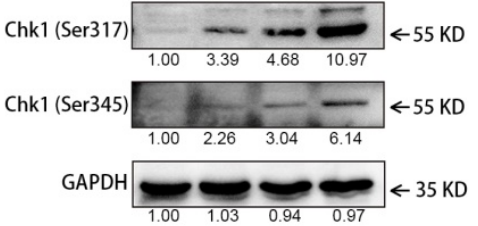

\section{D}

$\operatorname{ADR}(\mu \mathrm{M}) \quad \begin{array}{llll}0 & 0.5 & 1.0 & 2.0\end{array}$
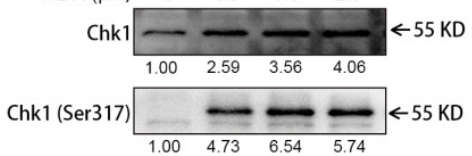

Chk1 (Ser345) $\longrightarrow-\square 5$ KD

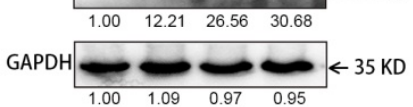

G

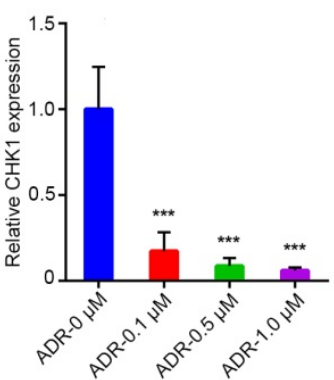

J $\operatorname{ADR}(\mu \mathrm{M})$
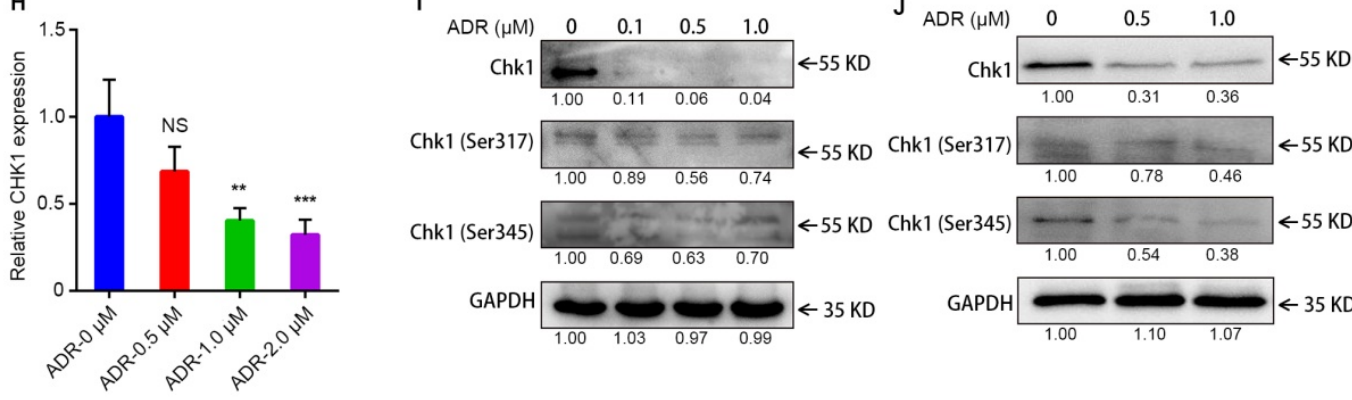

Chk1 (Ser317) \begin{tabular}{|llll}
\hline & & & \\
\hline
\end{tabular}

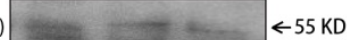

Chk1 (Ser345)

$\leftarrow 55$ KD Chk1 (Ser345)

GAPDH

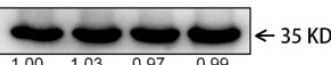

Figure 3. Activation of CHK1 by ADR depends on ER/PR status. A-C, G-H The mRNA level of CHK1 detected by RT-qPCR in MDA-MB-231 (A), MDA-MB-468 (B), MDA-MB-231/ADR (C), MCF-7 (G) and T47D (H) cells, with or without ADR (48 h; 0.5, 1, 2, 5 and 10 HM). D-F, I-J Protein levels of CHK1 in MDA-MB-231 (D), MDA-MB-468 (E), MDA-MB-231/ADR (F), MCF-7 (I) and T47D (J) cells, with or without ADR (48 h; 0.5, 1, 2, 5 and $10 \mu M)$, detected by Western blot. Data shown represent the means $( \pm$ $\mathrm{SD}$ ) of three independent experiments; $* P<0.05$, **P $<0.01$, ***P $<0.001$; NS, not significant; one-way ANOVA (A-C, G-H).

\section{References}

1. Xu W, Huang M, Guo J, Zhang H, Wang D, Liu T, Liu H, Chen S, Gao P, Mu K. The Role of CHK1 Varies with the Status of Oestrogen-receptor and Progesterone-receptor in the Targeted Therapy for Breast Cancer. International Journal of Biological Sciences. 2020; 16: 1388-1402. doi:10.7150/ijbs.41627. 